\title{
ASYMPTOTIC BEHAVIOR OF TEMPERATURE IN A NONLINEAR RADIATING, LINEAR ABSORBING ROD OF FINITE LENGTH*
}

\author{
By C. V. PAO (North Carolina State University)
}

1. Introduction. Consider the heat-conduction problem in a solid rod of length $l$ from which heat is absorbed or radiated along the rod. If the temperature distribution in the rod is initially known and if at one end of the rod energy absorbs (or radiates) linearly but radiates nonlinearly proportional to the $n$th power of the temperature $(n>1)$ while at the other end the temperature is kept at zero, then the temperature distribution $u(t, x)$ is governed by the following initial boundary-value problem:

$$
\begin{gathered}
u_{t}(t, x)=D u_{x x}(t, x)+c u(t, x) \quad(t>0,0<x<l), \\
u_{x}(t, 0)=\alpha u^{n}(t, 0)-\beta u(t, 0)-h(t) \quad(t>0), \\
u(t, l)=0 \\
u(0, x)=u_{0}(x) \quad(0 \leq x \leq l),
\end{gathered}
$$

where $D, c, \alpha, \beta$ are physical constants and $h, u_{0}$ are non-negative functions representing the boundary source and initial temperature, respectively. We assume that $u_{0}$ satisfies the boundary condition (1.2) at $t=0$ and the constants $D, \alpha$ are positive but allow $c$ and $\beta$ to be either positive or non-positive. Thus positive values of $c, \beta$ represent absorption of energy (heat) along the rod and at $x=0$, respectively, while negative values of $c, \beta$ represent radiation at the corresponding location.

The problem (1.1)-(1.3) and its various generalization have been treated by several authors (cf. [1-8]). The recent work by Keller-Olmstead [5] and Hartka [4] studies the asymptotic behavior of the temperature in a semi-infinite rod for the case $c \leq 0, u_{0} \equiv 0$. In view of the zero initial function, the temperature obtained in these papers is due solely to the boundary source $h(t)$ which is assumed to satisfy the condition $\int_{0}^{\infty} h(t) d t<\infty$. Several estimates for insuring the diminishing property of $u$ as $t \rightarrow \infty$ are given in $[3,5]$ for the case $c=\beta=0$ and in [4] for the case $\beta^{2}+c \leq 0$. However, when the rod is finite, no matter how great its length may be, it is reasonable to expect that the temperature remains diminishing to zero for certain range of values of $c, \beta$ even if both constants are positive. Furthermore, it is interesting to know whether $u(t, x)$ can diminish to zero if the boundary input accumulates unbounded, that is, if $h(t)$ satisfies the condition $\int_{0}^{\infty} h(t) d t=\infty$. The purpose of this paper is to study this physical expectation and to give some explicit estimates for the diminishing property of the solution. We also extend the above investigation to a heat-conduction problem in a two-dimensional rectangular region. The consideration of a finite domain instead of a semi-infinite domain makes it possible to sharpen the results obtained in [3-5] and leads to more information about the solution. In fact, we obtain explicit estimates for the rate of decay for the solution

* Received October 2, 1975; revised version received January 23, 1976. 
in terms of the physical parameters $D, c, \alpha, \beta, n, l$ and the boundary source $h(t)$. The inclusion of the constant $D$ in (1.1) is to exhibit the dependence of the asymptotic behavior of the solution on the thermal conductivity of the medium.

In order to investigate the asymptotic behavior of the temperature for a finite rod we apply a comparison theorem from a recent paper [10] by Pao in which upper and lower bounds of the temperature are given. The upper bound, called "upper solution", is required to satisfy only some inequalities in the system so that it gives considerable flexibility in the choice of such a function. (The lower bound is taken as zero for simplicity.) Hence to insure the asymptotic diminishing property of the solution it suffices to construct an upper solution having the desired diminishing property. It turns out that, depending on the values of $c, \beta$ and the asymptotic behavior of $h(t)$, we can construct explicit upper bounds for the solution of the problem (1.1)-(1.3) and for an immediate extension of a two-dimensional problem. As a matter of fact, our approach can be used to treat higher-dimensional domains and space-time dependent physical parameters. In this paper, however, we limit our discussion to the system (1.1)-(1.3) and its extension in the form of (3.1)-(3.3) in Sec. 3.

2. Asymptotic behavior of the solution for the rod problem. In this section we study the asymptotic behavior of the solution for the rod problem (1.1)-(1.3) by a suitable choice of an upper solution which is defined as follows:

Definition 2.1. A smooth non-negative function $\tilde{u}(t, x)$ is called an upper solution if it satisfies the following conditions:

$$
\begin{aligned}
\tilde{u}_{t}-D \tilde{u}_{x x}-c \tilde{u} \geq 0 & (t>0,0<x<l) \\
\tilde{u}_{x} \leq \alpha \tilde{u}^{n}-\beta \tilde{u}-h & (t>0, x=0) \\
\tilde{u}(t, l) \geq 0 & (t>0) \\
\tilde{u}(0, x) \geq u_{0}(x) & (0 \leq x \leq l) .
\end{aligned}
$$

By a smooth function we mean a continuous function on $[0, \infty) \times[0, l]$ whose first derivative in $t$ and second derivative in $x$ exist and are continuous in $(0, \infty) \times(0, l)$. The above definition shows that every solution of (1.1)-(1.3) is also an upper solution and the condition $\tilde{u}(t, l) \geq 0$ is trivially satisfied since $\tilde{u}$ is non-negative. An important implication of upper solutions is contained in the following.

Theorem 2.1. Let $\tilde{u}$ be an upper solution. Then there exists a unique non-negative solution to (1.1)-(1.3). Moreover,

$$
0 \leq u(t, x) \leq \tilde{u}(t, x) \quad(t \geq 0,0 \leq x \leq l) .
$$

The proof of the above theorem for a more general system was given in [10] by a monotone argument. We remark that in the present problem upper solutions do exist. In fact, if we denote by $\bar{u}_{0}, \bar{h}_{0}$ the respective least upper bounds of $u_{0}(x)$ and $h(t)$, and if $K_{0}$ is a constant satisfying

$$
\left.K_{0} \geq \max \left\{1, \bar{u}_{0},[\bar{h}+|\beta|) / \alpha\right]^{1 / n-1}\right\},
$$

then the function $\tilde{u}=K_{0} \exp (|c| t)$ is an upper solution. However, our aim is to construct some other upper solution so that it can be used to determine the diminishing property of the solution. It is obvious that the choice of $\tilde{u}$ depends on the values of $\beta, c$ and the 
asymptotic behavior of $h(t)$. Consider, for example, $h(t)=0(\exp (-\gamma t))$ as $t \rightarrow \infty$, where $\gamma>0$ is a constant. Then we may seek an upper solution in the form of $\tilde{u}=\exp (-\delta t) \phi(x)$, where $\delta$ is a positive constant and $\phi$ is a non-negative function on $[0, l]$. In view of Definition 2.1, it suffices to find a $\phi(x) \geq 0$ such that

$$
\begin{array}{rr}
D \phi_{x x}+(\delta+c) \phi \leq 0 \quad(0<x<l), \\
\exp (-\delta t) \phi_{x}(0) \leq \alpha \exp (-n \delta t)(\phi(0))^{n}-\beta \exp (-\delta t) \phi(0)-h(t) \quad(t>0), \\
\phi(x) \geq u_{0}(x) \quad(0 \leq x \leq l) .
\end{array}
$$

Assume that $c<D(\pi / 2 l)^{2}$. Then a possible choice of $\phi$ and $\delta$ is $\phi=K \cos (\pi x / 2 l)$ and $0<\delta<D(\pi / 2 l)^{2}-c$, where $K>0$ is to be determined. Clearly, $\phi(x) \geq 0$ on [0, l] and satisfies the first condition in (2.1). Since $\phi(0)=K, \phi_{x}(0)=0$ the second condition in $(2.1)$ is equivalent to

$$
\alpha K^{n} \exp (-n \delta t) \geq \beta K \exp (-\delta t)+h(t) \quad(t>0) .
$$

Now by the hypothesis $h(t)=0(\exp (-\gamma t))$ as $t \rightarrow \infty$, there exist positive constants $T$ and $K^{\prime}$ such that $h(t) \leq K^{\prime} \exp (-\gamma t)$ for $t>T$. Let $\bar{h}=\sup \{h(t) ; 0 \leq t \leq T\}$. Then (2.2) is satisfied if the constant $K$ satisfies the conditions

$$
\begin{aligned}
& \alpha K^{n} \geq \beta K \exp (\delta(n-1) t)+\bar{h} \exp (n \delta t) \quad(0 \leq t \leq T), \\
& \alpha K^{n} \geq \beta K \exp (\delta(n-1) t)+K^{\prime} \exp (-(\gamma-n \delta) t) \quad(t>T) .
\end{aligned}
$$

Since $n>1$ and $T$ is finite, the first inequality in (2.3) can be fulfilled by a sufficiently large value of $K$. To establish the second inequality we first consider the case $\beta \leq 0$. Then the second inequality in (2.3) is satisfied by choosing $\delta \leq \gamma / n$ and $K \geq\left(K^{\prime} / \alpha\right)^{1 / n}$. With this choice of $\delta$ and $K$ the function $\tilde{a}=K \exp (-\delta t) \cos (\pi x / 2 l)$ is an upper solution of (1.1)-(1.3) for any initial function $u_{0}(x) \leq K \cos (\pi x / 2 l)$. On the other hand, if for some $\gamma>0, h(t)=0\left((1+t)^{-\gamma}\right)$ or $h(t)=0\left(t^{-\gamma}\right)$ as $t \rightarrow \infty$. Then we seek an upper solution in the form of $\tilde{u}=(1+t)^{-\delta} \phi(x)$. In this case, the requirement on $\phi$ becomes

$$
\begin{gathered}
D \phi_{x x}+\left[(1+t)^{-1} \delta+c\right] \phi \leq 0 \quad(t>0,0<x<l), \\
(1+t)^{-\delta} \phi_{x}(0) \leq \alpha(1+t)^{-n \delta}(\phi(0))^{n}-\beta(1+t)^{-\delta} \phi(0)-h(t) \quad(t>0), \\
\phi(x) \geq u_{0}(x) \quad(0 \leq x \leq l) .
\end{gathered}
$$

If we choose $\phi$ as in the previous case, then $\phi \geq 0$ on $[0, l]$ and the first condition in (2.4) holds. The second condition reduces to

$$
\alpha K^{n}(1+t)^{-n \delta} \geq \beta K(1+t)^{-\delta}+h(t) \quad(t>0) .
$$

Now if $h(t)=0\left((1+t)^{-\gamma}\right)$ as $t \rightarrow \infty$, then there exist constants $T, K^{\prime}$ such that

$$
h(t) \leq K^{\prime}(1+t)^{-\gamma} \text { for } t>T .
$$

The inequality (2.6) also holds (except possibly with some different values of $T$ and $K^{\prime}$ ) if $h(t)=O\left(t^{-\gamma}\right)$ as $t \rightarrow \infty$. In any case the condition (2.5) is fulfilled if

$$
\begin{aligned}
& \alpha K^{n} \geq \beta K(1+t)^{(n-1) \delta}+\bar{h}(1+t)^{n \delta} \quad(0 \leq t \leq T), \\
& \alpha K^{n} \geq \beta K(1+t)^{(n-1) \delta}+K^{\prime}(1+t)^{-(\gamma-n \delta) t} \quad(t>T) .
\end{aligned}
$$


Clearly, the above inequalities hold for any $\delta, K$ satisfying

$$
0<\delta \leq \gamma / n, \quad K \geq \max \left\{(\bar{h} / \alpha)^{1 / n}(1+T)^{\delta}, \quad\left(K^{\prime} / \alpha\right)^{1 / n}\right\}
$$

when $\beta \leq 0$. With these values of $\delta, K$, the function $\tilde{u}=K(1+t)^{-\delta} \cos (\pi x / 2 l)$ is an upper solution for any $u_{0}(x) \leq K \cos (\pi x / 2 l)$. Hence for the case $\beta \leq 0$ we have the following conclusion:

Theorem 2.2. Let $c<D(\pi / 2 l)^{2}, \beta \leq 0$ and let $u$ be the solution of (1.1)-(1.3) with $u_{0}(x) \leq M_{0} \cos (\pi x / 2 l)$ for some constant $M_{0}$. Then there exist positive constants $K, T$ such that

$$
u(t, x) \leq K e^{-\delta} \cos (\pi x / 2 l) \text { for } t>T, \quad 0 \leq x \leq l
$$

when $h(t)=0(\exp (-\gamma t))$ as $t \rightarrow \infty(\gamma>0)$, and

$$
u(t, x) \leq K(1+t)^{-\delta t} \cos (\pi x / 2 l) \text { for } t>T, \quad 0 \leq x \leq l
$$

when $h(t)=0\left((1+t)^{-\gamma}\right)$ or $h(t)=0\left(t^{-\gamma}\right)$ as $t \rightarrow \infty$, where

$$
\delta=\min \left\{D(\pi / 2 l)^{2}-c, \gamma / n\right\} .
$$

Remark 2.1. It is readily seen that the result in (2.9) remains true if $h(t)=0\left((a+t)^{-\gamma}\right)$ as $t \rightarrow \infty$, where $a$ is any constant. For in this case the relation (2.6) still holds since

$$
h(t) \leq K(a+t)^{-\gamma}=K(1+t)^{-\gamma}[(1+t) /(a+t)]^{\gamma}, \quad(t>T)
$$

and $[(1+t) /(a+t)]^{\gamma}$ is bounded on $[T, \infty)$ for any constant $a>-T$. In the special case of $h(t)=0$ on $\left[T_{1}, \infty\right)$ for some $T_{1}$ as assumed in [4], all the conditions on $h$ in Theorem 3.1 are satisfied for any $\gamma>0$. In this situation, the temperature decays to zero exponentially with a decay rate $\delta=D(\pi / 2 l)^{2}-c$. On the other hand, the work in [5] requires that $\int_{0}^{\infty} h(t) d t<\infty$. However, the class of functions $h(t)$ in the form $K_{1}(a+t)^{-\gamma}$ with $0<\gamma \leq 1$ satisfies the requirement in Theorem 2.2 but $\int_{0}^{\infty} h(t) d t=\infty$. Hence our results show that in a finite rod the temperature can decay to zero even if the energy input $h(t)$ accumulates unboundedly and linear absorption occurs along the rod. In other words, the combined energy radiation at the end $x=0$ and the "leakage" (of heat) at $x=l$ dominate the energy absorption due to the simultaneous input $h$ and the absorption along the surface of the rod. Hence the consideration of a finite rod instead of a semi-infinite rod leads to sharper results than those obtained in $[4,5]$.

We next consider the case where linear absorption may occur at the boundary $x=0$ (i.e., $\beta>0)$. Assume, as usual, that $c<D(\pi / 2 t)^{2}$ and $h(t)=0(\exp (-\gamma t))($ or $h(t)=$ $\left.0\left((a+t)^{-\gamma}\right)\right)$. Then we seek an upper solution in the same form $\tilde{u}=\exp (-\delta t) \phi(x)$, where $\delta>0$ is to be chosen. The function $\phi$ must be non-negative and satisfy the conditions in (2.1). As a candidate for this function we take

$$
\phi_{\lambda}(x)=K(\cos \lambda x-(\beta / \lambda) \sin \lambda x),
$$

where $K, \lambda$ are some positive constants with $\lambda \leq(\pi / 2 l)$. Then $\phi_{\lambda}(x) \geq 0$ if and only if

$$
\tan \lambda x \leq \lambda / \beta \text { for } 0 \leq \lambda x \leq \pi / 2 .
$$

Since $\tan \lambda x$ is an increasing function of $\lambda x$ for $0 \leq \lambda x \leq \pi / 2$, it suffices to choose a $\lambda$ such that

$$
\tan \lambda l \leq \lambda / \beta=(\lambda l) /(\beta l) .
$$


Now if $\beta l<1$ then there exists a positive number $\lambda_{0} \leq(\pi / 2 l)$ such that $\tan \lambda_{0} l=$ $\left(\lambda_{0} l\right) /(\beta l)$. With $\lambda=\lambda_{0}$ we then have $\tan \lambda_{0} x \leq \tan \lambda_{0} l=\lambda_{0} / \beta$ for $0 \leq \lambda_{0} x \leq \pi / 2$, and thus the function $\phi_{\lambda_{0}}$ given by (2.11) with $\lambda=\lambda_{0}$ is non-negative on $[0, l]$. To show that $\boldsymbol{\phi}_{\lambda_{0}}$ satisfies the conditions in (2.1), we observe from the non-negative property of $\phi_{\lambda_{0}}$ that the first condition is fulfilled for any $\delta \leq D \lambda_{0}{ }^{2}-c$. Since $\phi_{\lambda_{0}}(0)=K$ and $\left(\phi_{\lambda_{0}}\right)_{x}(0)+$ $\beta \boldsymbol{\phi}_{\lambda_{0}}(0)=0$, the second condition in (2.1) is also satisfied for any $\delta \leq \gamma / n$ and a sufficiently large $K$. Hence if we choose

$$
\delta=\min \left\{D{\lambda_{0}}^{2}-c, \gamma / n\right\},
$$

then the function

$$
\tilde{u}=\exp (-\delta t) \boldsymbol{\phi}_{\lambda_{0}}(x) \equiv K \exp (-\delta t)\left(\cos \lambda_{0} x-\left(\beta / \lambda_{0}\right) \sin \lambda_{0} x\right)
$$

is an upper solution of (1.1)-(1.3) for every $u_{0} \leq \phi_{\lambda_{0}}$. In the case of $h(t)=0\left(t^{-\alpha}\right)$ or, more generally, $h(t)=0\left((a+t)^{-\gamma}\right)$ as $t \rightarrow \infty$, the same argument given above leads to an upper solution in the form of $(1+t)^{-\delta} \phi_{\lambda_{0}}(x)$, where $\delta$ and $\lambda_{0}$ are the same as in the previous case. In conclusion, we obtain the following

Theorem 2.3. Let $c<D(\pi / 2 l)^{2}, \beta<l^{-1}$ and let $u$ be the solution of (1.1)-(1.3) with $u_{0}(x) \leq \phi_{\lambda_{0}}(x)$, where $\phi_{\lambda_{0}}(x)$ is given by (2.11). Then there exists a constant $T$ such that

$$
u(t, x) \leq \exp (-\delta t) \phi_{\lambda_{\circ}}(x) \text { for } t>T, \quad 0 \leq x \leq l
$$

when $h(t)=0(\exp (-\gamma t))$ as $t \rightarrow \infty$, and

$$
u(t, x) \leq(1+t)^{-\delta t} \phi_{\lambda_{0}}(x) \text { for } t>T, \quad 0 \leq x \leq l
$$

when $h(t)=0\left((a+t)^{-\gamma}\right)$ as $t \rightarrow \infty$, where $a$ is any real number and $\delta$ is the decay constant given by (2.14).

Remark 2.2. The requirement $u_{0} \leq \phi_{\lambda_{0}}$ in Theorem 2.3 is not very restrictive since the value of $K$ in (2.11) can be chosen arbitrarily large. Notice that the value of $c$ in Theorems 2.2 and 2.3 is allowed to be negative or zero. In this case, the requirement on $c$ in these theorems is automatically satisfied and the value of $\delta$ may be improved.

3. An extension to a two-dimensional problem. The techniques developed in the previous sections can be extended to some heat-conduction problems in multidimensional spatial domains. For simplicity, we limit our discussion to a simple two-dimensional model which is an immediate extension of the rod problem.

Consider the system

$$
\begin{gathered}
u_{t}=D\left(u_{x x}+u_{y y}\right)+c u \quad(t>0,(x, y) \in R), \\
u_{x}(t, 0, y)=\alpha(u(t, 0, y))^{n}-\beta u(t, 0, y)-h(t, y) \quad(t>0,0<y<b), \\
u(t, a, y)=u(t, x, 0)=u(t, x, b)=0 \quad(t \geq 0,0<x<a, 0<y<b), \\
u(0, x, y)=u_{0}(x, y) \quad(x, y \in \bar{R})
\end{gathered}
$$

where $R=\{(x, y) ; 0<x<a, 0<y<b\}$ and $h, u_{0}$ are non-negative continuous functions. We assume that $u_{0}$ satisfies the boundary condition (3.2) at $t=0$. The above system describes the heat-conduction in a rectangular region with energy being absorbed $(c>0)$ or radiated $(c<0)$ over its lateral surface. Our consideration of this particular model provides a simple comparison with the one-dimensional rod problem. 
Definition 3.1. A smooth non-negative function $\tilde{u}$ is called an upper solution if it satisfies

$$
\begin{aligned}
\tilde{u}_{t} & \geq D\left(\tilde{u}_{x x}+\tilde{u}_{\nu \nu}\right)+c \tilde{u}, \\
\tilde{u}_{x}(t, 0, y) & \leq \alpha(\tilde{u}(t, 0, y))^{n}-\beta \tilde{u}(t, 0, y)-h(t, y), \\
\tilde{u}(t, a, y) & \geq 0, \quad \tilde{u}(t, x, 0) \geq 0, \quad \tilde{u}(t, x, b) \geq 0, \\
\tilde{u}(0, x, y) & \geq u_{0}(x, y) .
\end{aligned}
$$

It is easily seen that upper solutions exist and can be explicitly given (e.g., see Eqs. (3.7), (3.13)). By a theorem in [10] the problem (3.1)-(3.3) has a unique non-negative solution $u$ which satisfies the relation

$$
0 \leq u(t, x, y) \leq \tilde{u}(t, x, y) \quad(t \geq 0,0 \leq x \leq a, 0 \leq y \leq b)
$$

for any upper solution $\tilde{u}$. Hence an upper estimate for the asymptotic behavior of the solution can be obtained from the asymptotic property of a suitable upper solution. A convenient choice of such a function is given by $\tilde{u}=\exp (-\delta t) \phi(x, y)$ when $h(t, y)=$ $O(\exp (-\gamma t))$, and $\tilde{u}=(1+t)^{-\delta} \phi(x, y)$ when $h(t, y)=O\left((a+t)^{-r}\right)$. In the form of $\exp (-\delta t) \phi, \tilde{u}$ becomes an upper solution if $\phi$ is non-negative on $\bar{R}$ and satisfies the following conditions:

$$
\begin{gathered}
D\left(\phi_{x x}+\phi_{y y}\right)+(\delta+c) \phi \leq 0, \\
\phi_{x}(0, y) \leq \alpha \exp (-(n-1) \delta t)(\phi(0, y))^{n}-\beta \phi(0, y)-\exp (\delta t) h(t, y), \\
u_{0}(x, y) \leq \phi(x, y) .
\end{gathered}
$$

Suppose that $c<D\left[(\pi / 2 a)^{2}+(\pi / 4 b)^{2}\right]$. Then we choose $\delta \leq D\left[(\pi / 2 a)^{2}+(\pi / 4 b)^{2}\right]-c$ and

$$
\phi(x, y)=K \cos (\pi x / 2 a) \sin (\pi(y+b) / 4 b) .
$$

Clearly, $\phi$ is non-negative on $\bar{R}$ and satisfies the first condition in (3.6) for any constant $K>0$. To insure the second condition it suffices to show that

$\alpha[K \sin (\pi(y+b) / 4 b)]^{n} \geq \beta \exp ((n-1) \delta t)[K \sin (\pi(y+b) / 4 b)]+\exp (n \delta t) h(t, y)$

for some positive constants $\delta$ and $K$. Consider the case where $\beta \leq 0$ and $h(t, y)$ $=0(\exp (-\gamma t))$ as $t \rightarrow \infty$ (uniformly in $y)$. Then there exist constants $K^{\prime}, T$ such that $h(t, y) \leq K^{\prime} \exp (-\gamma t)$ for $t>T, 0 \leq y \leq b$. In view of $\sin (\pi(y+b) / 4 b) \geq 1 / \sqrt{ } 2$ on $[0, b]$ the relation (3.8) holds if we choose $\delta \leq \gamma / n$ and $K$ such that

$$
\alpha(K / \sqrt{ } 2)^{n} \geq \max \left\{\exp (n \delta T) \bar{h}, K^{\prime}\right\},
$$

where $\bar{h}=\sup \{h(t, y) ; 0 \leq t \leq T, 0 \leq y \leq b\}$. This shows that for any $u_{0}(x, y) \leq \phi(x, y)$ the function $\tilde{u}=\exp (-\delta t) \phi$ is an upper solution. The same argument shows that $\tilde{u} \equiv(1+t)^{-\delta} \phi$ is an upper solution when $h(t, y)=0\left(\left(a_{1}+t\right)^{-\delta}\right)$ uniformly in $y$ as $t \rightarrow \infty$, where $\phi$ is given by (3.7) except with a possibly different value of $K$. This observation leads to the following conclusion:

Theorem 3.1. Let $\beta \leq 0, c<D\left[(\pi / 2 a)^{2}+(\pi / 4 b)^{2}\right]$ and let $u$ be the solution of (3.1)-(3.3) with $u_{0} \leq \phi$, where $\phi$ is given by (3.7). Then there exists a constant $T$ such that

$$
u(t, x, y) \leq \exp (-\delta t) \phi(x, y) \quad \text { for } t>T, \quad(x, y \in \bar{R}),
$$

when $h(t, y)=O\left(e^{-\gamma t}\right)$ uniformly in $y$ as $t \rightarrow \infty$; and

$$
u(t, x, y) \leq(1+t)^{-\delta} \phi(x, t) \quad \text { for } t>T, \quad(x, y) \in \bar{R}
$$


when $h(t, y)=0\left(\left(a_{1}+t\right)^{-\gamma}\right)$ uniformly in $y$ as $t \rightarrow \infty$, where

$$
\delta=\min \left\{\gamma / n, D\left((\pi / 2 a)^{2}+(\pi / 4 b)^{2}\right)-c\right\} .
$$

In particular, if for some $T_{1}<\infty, h(t, y)=0$ for $t>T_{1}, 0 \leq y \leq b$ then (3.10) holds with $\delta=D\left((\pi / 2 a)^{2}+(\pi / 4 b)^{2}\right)-c$.

The result in Theorem 3.1 shows that if $h(t, y)=0(\exp (-\gamma t))$ for some large $\gamma$ or if $h(t, y)=0$ for sufficiently large $t$, then the temperature decays exponentially with a decay constant $\delta=D\left[(\pi / 2 a)^{2}+(\pi / 4 b)^{2}\right]-c$ which is larger than the corresponding decay constant in the rod problem by the amount $D(\pi / 4 b)^{2}$. Physically, this is to be expected since energy leaks through the boundary $y=0$ and $y=b$. Notice that if energy radiates from the surface of the plate the value of $c$ is negative and thus the temperature decays even faster.

When linear absorption occurs at the boundary $x=0$ (i.e., $\beta>0$ ) we choose the function $\phi$ in the form

$$
\phi_{\lambda}(x . y)=K(\cos \lambda x-(\beta / \lambda) \sin \lambda x) \sin (\pi(y+b) / 4 b) .
$$

If we use $\lambda=\lambda_{0}$ and the same value of $K$ in the rod problem (with $l$ replaced by $a$ ), the same argument as for the one-dimensional rod problem leads to the following:

Theorem 3.2. Let $\beta<a^{-1}, c<D\left[(\pi / 2 a)^{2}+(\pi / 4 b)^{2}\right]$ and let $u$ be the solution of (3.1)-(3.3). Then for any initial function $u_{0}(x, y) \leq \phi_{\lambda_{0}}(x, y)$, where $\phi_{\lambda_{0}}$ is given by (3.13), $u$ satisfies the estimates (3.10), (3.11) in Theorem 3.1 except with the decay constant $\delta$ given by

$$
\delta=\min \left\{D\left(\lambda_{0}^{2}+(\pi / 4 b)^{2}-c\right), \gamma / n\right\},
$$

where $\lambda_{0}$ is determined from the equation $\tan \lambda a=\lambda / \beta$.

Proof. It suffices to show that $\phi_{\lambda_{0}}$ satisfies the second condition in (3.6). But in view of the choice of $K$ and $\lambda_{0}$, this condition follows from the same argument as for the rod problem. We omit the details.

\section{REFERENCES}

[1] C. Y. Chan, Positive solutions for nonlinear parabolic second initial boundary-value problem, Quart. Appl. Math. 31, 443-454 (1974)

[2] A. Friedman, Generalized heat transfer between solids and gases under nontinear boundary conditions, J. Math. Mech. 8, 161-183 (1959)

[3] R. A. Handelsman and W. E. Olmstead, Asymptotic solution to a class of nonlinear Volterra integral equations, SIAM J. Appl. Math. 22, 373-384 (1972)

[4] J. E. Hartka, Temperature of a semi-infinite rod which radiates both linearly and nonlinearly, Quart. Appl. Math. 32, 101-111 (1974)

[5] J. B. Keller and W. E. Olmstead, Temperature of a nonlinearly radiating semi-linear soiid, Quart. Appl. Math. 29, 559-566 (1972)

[6] H. A. Levine and L. E. Payne, Non-existence theorem for the heat equation with nonlinear boundary conditions and for the porous medium equation backward in time, J. Diff. Eqns. 16, 319-334 (1974)

[7] W. R. Mann and F. Wolf, Heat transfer between solids and gases under nonlinear boundary conditions, Quart. Appl. Math. 9, 163-184 (1951)

[8] K. Padmavally, On a nonlinear integral equation, J. Math. Mech. 7, 533-555 (1958)

[9] C. V. Pao, Successive approximations of some nonlinear initial boundary-value problems, SIAM J. Math. Anal. 5, 91-102 (1974)

[10] C. V. Pao, Positive solutions of a nonlinear boundary-value problem of parabolic type, J. Diff. Eqns. 22, 145-163 (1976)

[11] M. H. Protter and H. F. Weinberger, Maximum principles in differential equations, Prentice-Hall, Fnglewood Cliffs, 1967 